\title{
STUDIES ON THE MECHANISM OF THE HYAMINE- INDUCED ACROSOME REACTION IN EJACULATED BOVINE SPERMATOZOA
}

\author{
F. B. P. WOODING \\ Agricultural Research Council Institute of Animal Physiology, \\ Babraham, Cambridge CB2 $4 A T$
}

A basic requirement for membrane fusion is that the two membranes must be brought sufficiently close together to allow fusion. This is complicated by the fact that most intracellular membranes have the same overall negative charge, at least when isolated. It has been pointed out that on theoretical grounds there is less of an energy barrier to fusion if at least one of the membranes is distorted to form a narrow protrusion (Poste \& Allison, 1973). Most fusion reactions occur rapidly and their exact site is difficult to predict. In this respect the acrosome reaction of spermatozoa has the advantage that there is a large area of membranes between which fusion occurs. This electron microscope study of that fusion process is part of a wider investigation (Wooding \& O'Donnell, 1971; Wooding, 1973) to see whether the complex sequence of changes in sperm membranes before fertilization that has been described in other mammals (Bedford, 1972, for a review) can be produced in the bull spermatozoon in vitro by detergents and other media.

The fusion of the acrosome outer membrane with the plasmalemma is the basis of the acrosome reaction in both vertebrate and invertebrate spermatozoa (Dan, 1970; Bedford, 1972, for review). In mammals the reaction allows release of the acrosomal enzymes required to achieve passage of the spermatozoon through the investments surrounding the oocyte.

In several mammals the acrosome reaction does not occur until the inseminated spermatozoon has spent a certain time in the female reproductive tract or has been incubated in vitro in one of several media. This preliminary incubation, which does not appear to change the fine structure of the spermatozoon significantly, signifies the need for capacitation (Austin, 1967; Bedford, 1972, for reviews). The time required for capacitation in vitro or in vivo is very variable under different conditions and in different animals. Initial membrane changes in the acrosome reaction are therefore difficult to study since they cannot be accurately predicted.

The capacitation requirement can be bypassed, however, by using the detergent Hyamine to induce the acrosome reaction. Hyamine was introduced by Hartree \& Srivastava (1965) to facilitate the release of the acrosomal enzymes from spermatozoa and has been widely used for this purpose subsequently. It is usually employed at concentrations between 0.05 and $1 \%$ for incubations of spermatozoa for 30 to $90 \mathrm{~min}$ at $37^{\circ} \mathrm{C}$ (see Multamaki \& Pelliniemi, 1973, for references). Under these conditions very few membranes 
are left on the sperm head after incubation, but a few spermatozoa show a vesiculated outer acrosomal membrane (O'Donnell, Symons \& Wooding, 1970; Multamaki \& Pelliniemi, 1973). If the Hyamine concentration is reduced and the incubation shortened, up to $30 \%$ of the spermatozoa show vesiculation between the outer acrosomal membrane and the plasmalemma, with the remainder of the sperm membranes unaffected. Such spermatozoa resemble morphologically those found close to the egg surface which have undergone the acrosome reaction in vivo (Barros, Bedford, Franklin \& Austin, 1967; Bedford, 1972, for review) and also those showing vesiculation after treatment with bovine uterine fluid in vitro (see below). A small percentage of aged spermatozoa show a similar vesiculation of the acrosome outer membrane, the 'false acrosome reaction', but such spermatozoa do not possess any plasmalemma over the remainder of the sperm head (Bedford, 1970). Therefore the vesiculation induced by a brief treatment with low concentrations of Hyamine is considered to represent an acrosome reaction analogous to that which occurs in vivo. Hyamine is the best reagent so far reported; it has the ability to induce the reaction without considerable prior incubation of ruminant spermatozoa (Hartree \& Srivastava, 1965). Triton X100 removes bovine sperm membranes in a reproducible sequence but does not induce any vesiculation (Wooding, 1973).

Most observations on the sperm acrosome reaction have been made at the light microscope level. Vesiculation without prior capacitation has been reported for rabbit spermatozoa by Gabara, Gledhill, Croce, Cesarini \& Koprowski (1973) using lysolecithin at $600 \mu \mathrm{g} / \mathrm{ml}$, but no details of the process were given. Buthala, Ericsson \& Chubb (1971) found that absorption of Sendai virus could bypass the need for capacitation in the rabbit, but it is not clear whether the acrosome reaction occurs under these conditions, since the microscopy was of whole-mount preparations of spermatozoa. The Hyamine effect has been studied by adding the twice-washed ejaculated spermatozoa to detergent made up to 0.005 to $0.01 \%$ in the solution containing salts and fructose in which the spermatozoa were washed (O'Donnell, 1969). Samples were pipetted out of the solution into glutaraldehyde fixative to stop the detergent action. The spermatozoa were pelleted and embedded in Araldite, and 200 to 300 sperm heads were counted from sections of each sample. Three categories were distinguished: spermatozoa with an intact plasmalemma and unswollen acrosome (intact spermatozoa), spermatozoa with a vesiculated upper acrosomal membrane and an intact plasmalemma starting from the equatorial segment of the acrosome (a.r. spermatozoa), and spermatozoa with no plasmalemma over the head and a swollen acrosomal boundary (swollen spermatozoa). Table 1 shows the results of a typical experiment. The lowest concentration which consistently gives the acrosome reaction is $0.01 \%$; if the reaction occurs at all in concentrations below $0.01 \%, 20$ to $30 \%$ a.r. spermatozoa are still produced. Concentrations greater than $0.01 \%$ may produce an acrosome reaction, but they also remove most of the sperm plasmalemma and no intact spermatozoa survive. Since the object was to examine the onset of the acrosome reaction, only samples with some intact spermatozoa were examined in detail for evidence of the membrane changes. 
Morphological effects of Hyamine

The structure of the bovine sperm head has been amply documented at the electron microscope level. In an intact spermatozoon (Pl. 3, Fig. 8), the acrosome content is homogeneous and the plasmalemma intact. The gap between the plasmalemma and the outer acrosome membrane varies considerably within any one sample. The percentage of intact spermatozoa ranged from 50 to $95 \%$ in different unwashed ejaculates from Jersey bulls, washing having no significant effect on either the percentage intact or the gap between acrosome and plasmalemma (Wooding \& O'Donnell, 1971). The remainder of the spermatozoa had swollen acrosomes and no plasmalemma over the head region. Less than $1 \%$ of untreated spermatozoa showed any vesiculation of the acrosome.

Table 1. Effect of incubation with or without Hyamine on bovine sperm membranes

\begin{tabular}{|c|c|c|c|c|c|c|}
\hline \multirow{2}{*}{$\begin{array}{c}\text { Time of } \\
\text { incubation at } \\
37^{\circ} \mathrm{C}\end{array}$} & \multicolumn{3}{|c|}{$\begin{array}{c}\text { Spermatozoa in } 0.01 \% \\
\text { Hyamine }(\%)\end{array}$} & \multicolumn{3}{|c|}{$\begin{array}{c}\text { Spermatozoa without } \\
\text { Hyamine }(\%)\end{array}$} \\
\hline & Intact & a.r. ${ }^{*}$ & Swollen & Intact & a.r.* & Swollen \\
\hline $\begin{array}{c}15 \mathrm{sec} \\
1 \mathrm{~min} \\
10 \mathrm{~min} \\
30 \mathrm{~min} \\
75 \mathrm{~min}\end{array}$ & $\begin{array}{l}35 \\
35 \\
38 \\
28 \\
29\end{array}$ & $\begin{array}{l}18 \\
19 \\
21 \\
26 \\
24\end{array}$ & $\begin{array}{l}47 \\
46 \\
41 \\
46 \\
47\end{array}$ & $\begin{array}{l}77 \\
60 \\
66 \\
53 \\
37\end{array}$ & $\begin{array}{l}0 \\
0 \\
1 \\
2 \\
8\end{array}$ & $\begin{array}{l}23 \\
40 \\
32 \\
44 \\
55\end{array}$ \\
\hline
\end{tabular}

* Spermatozoa with complete or partial acrosome reaction.

Incubation of the spermatozoa with $0.1 \%$ Hyamine for $90 \mathrm{~min}$ at $37^{\circ} \mathrm{C}$ results in a loss of both plasmalemma and outer acrosomal membrane in more than $90 \%$ of the sperm heads (Pl. 1, Fig. 1). Fragments of acrosome outer membrane which may or may not show vesiculation are present occasionally around the head and scattered in the pellet with the spermatozoa (Pl. 1, Fig. 1). After $1 \mathrm{~min}$ of $0.01 \%$ Hyamine, 20 to $30 \%$ of the spermatozoa show the vesiculation between plasmalemma and outer acrosomal membrane characteristic of the acrosome reaction (Table 1). The acrosomal content is reduced to a meshwork of fuzzy material on which the vesicles are held and a dark crescent of denser substance in the tip of the acrosome. The plasmalemma over the posterior part of the sperm head is continuous with the upper margin of the outer membrane of the equatorial segment of the acrosome (Pl. 1, Figs 2, 3 and 4; Pl. 2, Fig. 5).

Further incubation up to $120 \mathrm{~min}$ does not significantly alter the number of a.r. spermatozoa (Table 1) but there is a loss of plasmalemma over the posterior part of the spermatozoon, and up to $5 \%$ lose all trace of outer acrosomal membrane.

About 30 to $40 \%$ of spermatozoa are intact after short incubation times in $0.01 \%$ Hyamine, 40 to $45 \%$ have swollen acrosomes with a fuzzy outline and containing a dark apical crescent with no trace of plasmalemma (PI. 1, Fig. 3) and the remaining 20 to $30 \%$ show the a.r. The vesicles formed by the acro- 


\section{EXPLANATION OF PLATES}

\section{PLATE 1}

Fic. 1. Spermatozoa treated with $0.1 \%$ Hyamine for $90 \mathrm{~min}$ at $37^{\circ} \mathrm{C}$. No plasmalemma remains on the sperm heads or tails. The acrosome equatorial segment membranes (single arrow) and the inner acrosome membrane (double arrow) are present but considerably altered and non-continuous. The profile consisting of a string of vesicles (X) probably represents the remnants of the vesiculation between the original plasmalem$\mathrm{ma}$ and the anterior outer acrosomal membrane. $\times 15,550$.

Figs 2, 3 and 4. Spermatozoa treated with $0.01 \%$ Hyamine for $1 \mathrm{~min}$ at $37^{\circ} \mathrm{C}$. In Fig. 2 less density has been lost from the acrosome than in the spermatozoon showing vesiculation in Fig. 3. Fig. 2 presumably represents an earlier stage in the vesiculation process. The vesiculation does not occur over the equatorial segment of the acrosome (arrows, Figs 2 and 3 ) although the plasmalemma may be very close to the equatorial segment membrane as can be seen in Fig. 4 (a detail from Fig. 3). Notice the three classes of sperm morphology: intact (A), acrosome reacted (B) and swollen (C). Fig. 2, $\times 32,000$; Fig. 3, $\times 19,500$; Fig. $4, \times 105,000$.

\section{PLATE 2}

Figs 5, 6 and 7. Spermatozoa treated with $0.01 \%$ Hyamine for $1 \mathrm{~min}$ at $37^{\circ} \mathrm{C}$. In Fig. 5, a transverse section of part of a sperm head, note the fuzzy material connecting the vesicles, the range of size of the vesicles and the continuity of the inner acrosomal membrane (arrow). Fig. 6 shows a section including part of the surface of a vesiculated spermatozoon. The post-acrosomal sheath (single arrow) and the acrosome equatorial segment (double arrow) appear unaffected. The anterior acrosomal membrane has clearly vesiculated rather than forming a tubular meshwork, although occasional indications of structures more complex than a simple vesicle are seen (arrow, Fig. 7). Fig. 5, $\times 61,000$; Fig. 6, $\times 16,000$; Fig. 7, $\times 42,000$ (detail of Fig. 6).

\section{PLATE 3}

Fics 8 and 9. Washed spermatozoa, not treated with Hyamine. Fig. 8 shows a spermatozoon typical of 80 to $90 \%$ of the population with a plasmalemma widely separated from the acrosome. The spermatozoon in Fig. 9 has a closely adherent plasmalemma and represents about $5 \%$ of the total sample. $\times 17,000$.

Fics 10,11 and 12 . Spermatozoa treated with $0.01 \%$ Hyamine for $1 \mathrm{~min}$ at $37^{\circ} \mathrm{C}$. Fig. 10 shows a spermatozoon with the small amplitude ruffing of the plasmalemma. These are not found after Hyamine incubation times of more than $3 \mathrm{~min}$. There is no ruffling over the equatorial segment (arrow, Fig. 10). Higher magnification of similar ruffling shows that the plasmalemma may come as close to the outer acrosome membrane as 200 to 300 $\mathrm{nm}$ (Fig. 11) or even to a point contact (arrows, Fig. 12). Fig. 10, $\times 37,000 ;$ Fig. 11, $\times 150,000$; Fig. 12, $\times 150,000$.

\section{PLATE 4}

Fig. 13. Spermatozoon treated with $0.01 \%$ Hyamine for 1 min at $37^{\circ} \mathrm{C}$. Note the loss of density and slight swelling over the anterior part of the acrosome. Ruffling of the plasmalemma can be seen over the altered part of the acrosome but not over the equatorial segment (arrow). $\times 30,000$.

Figs 14 and 15. Spermatozoon treated with $0.01 \%$ Hyamine for $1 \mathrm{~min}$ at $37^{\circ} \mathrm{C}$. Note the partial vesiculation between the ruffled plasmalemma and the underlying outer acrosomal membrane. The acrosome content shows a considerable loss of density and is slightly swollen. Fig. 14, × 46,000; Fig. 15, $\times 155,000$ (detail of Fig. 14).

\section{PLATE 5}

FIGs 16,17 and 18. Spermatozoa treated with $0.01 \%$ Hyamine for $1 \mathrm{~min}$ at $37^{\circ} \mathrm{C}$. Such partly vesiculated spermatozoa are only found after Hyamine incubation times of less than $3 \mathrm{~min}$. The spermatozoa show no vesiculation over the equatorial segment, a variable loss of acrosome content and a ruffling of the outer acrosomal membrane rather than of the plasmalemma. Fig. 17 also shows an intact spermatozoon (arrow) with a small amplitude plasmalemma ruffling. Fig. 16, $\times 30,000 ;$ Fig. 17, $\times 19,500$; Fig. 18, $\times 31,000$.

Fias 19 and 20. Spermatozoa incubated in bovine uterine fluid for $3 \mathrm{hr}$ at $37^{\circ} \mathrm{C}$. The spermatozoon shows vesiculation restricted to the upper part of the acrosome. The plasmalemma is intact over the remainder of the sperm head and continuous with the upper margin of the equatorial segment (arrow, Fig. 20). Compare with B in Fig. 3. Fig. 19, $\times 28,000$; Fig. 20, $\times 61,000$ (detail of Fig. 19). 
PLATE 1

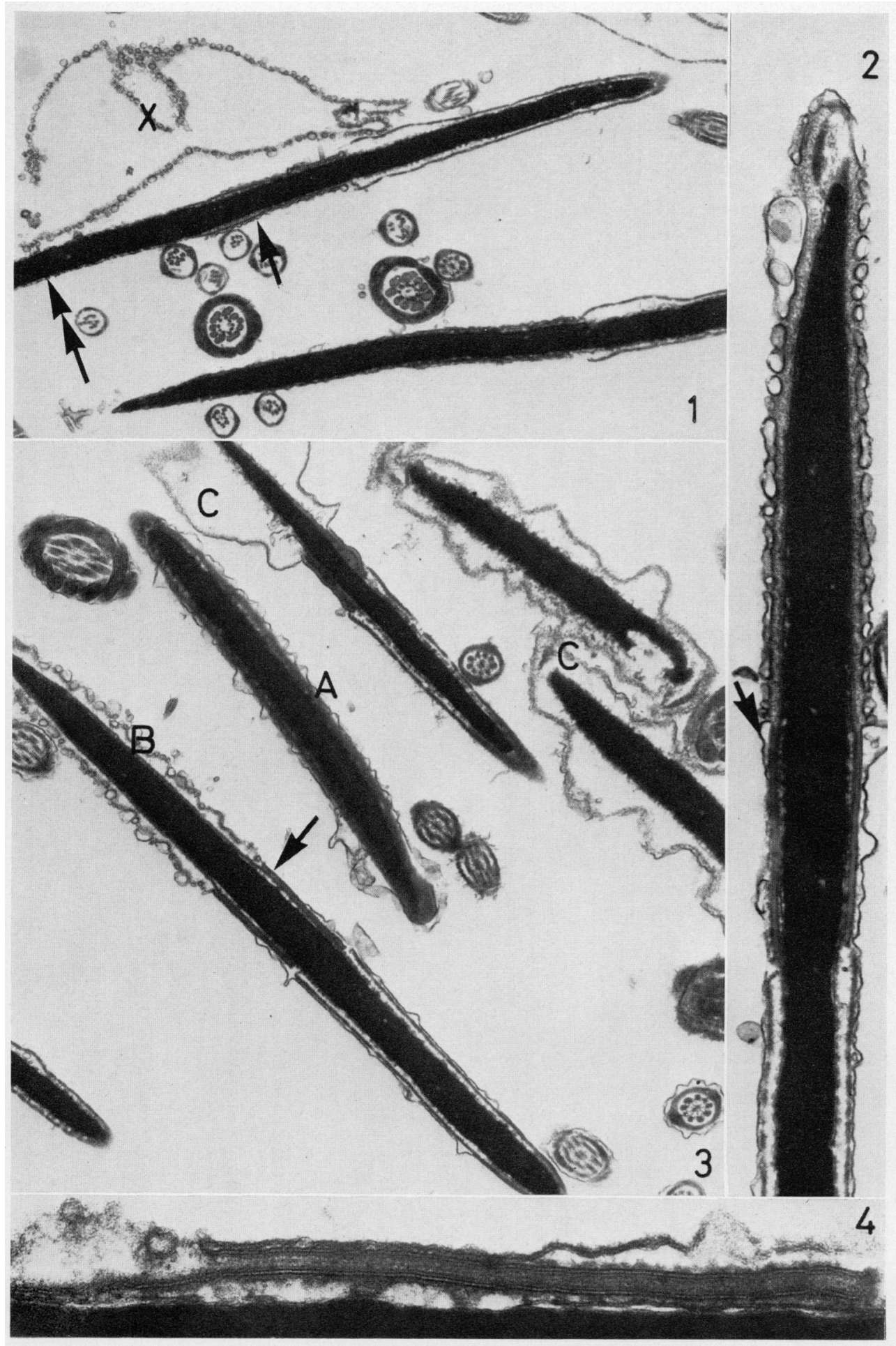

(Facing p. 188) 
PLATE ?

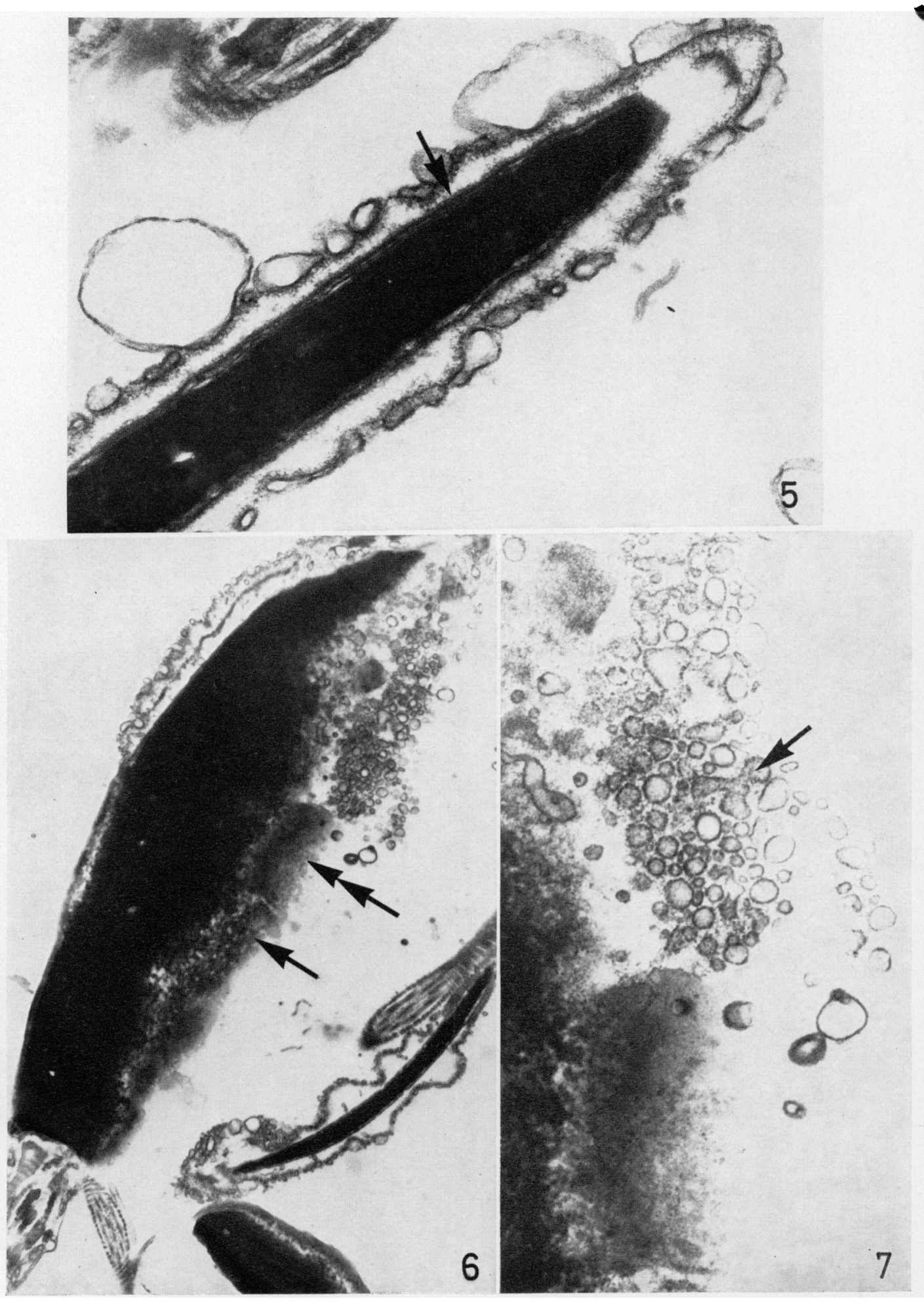


PIATE 3

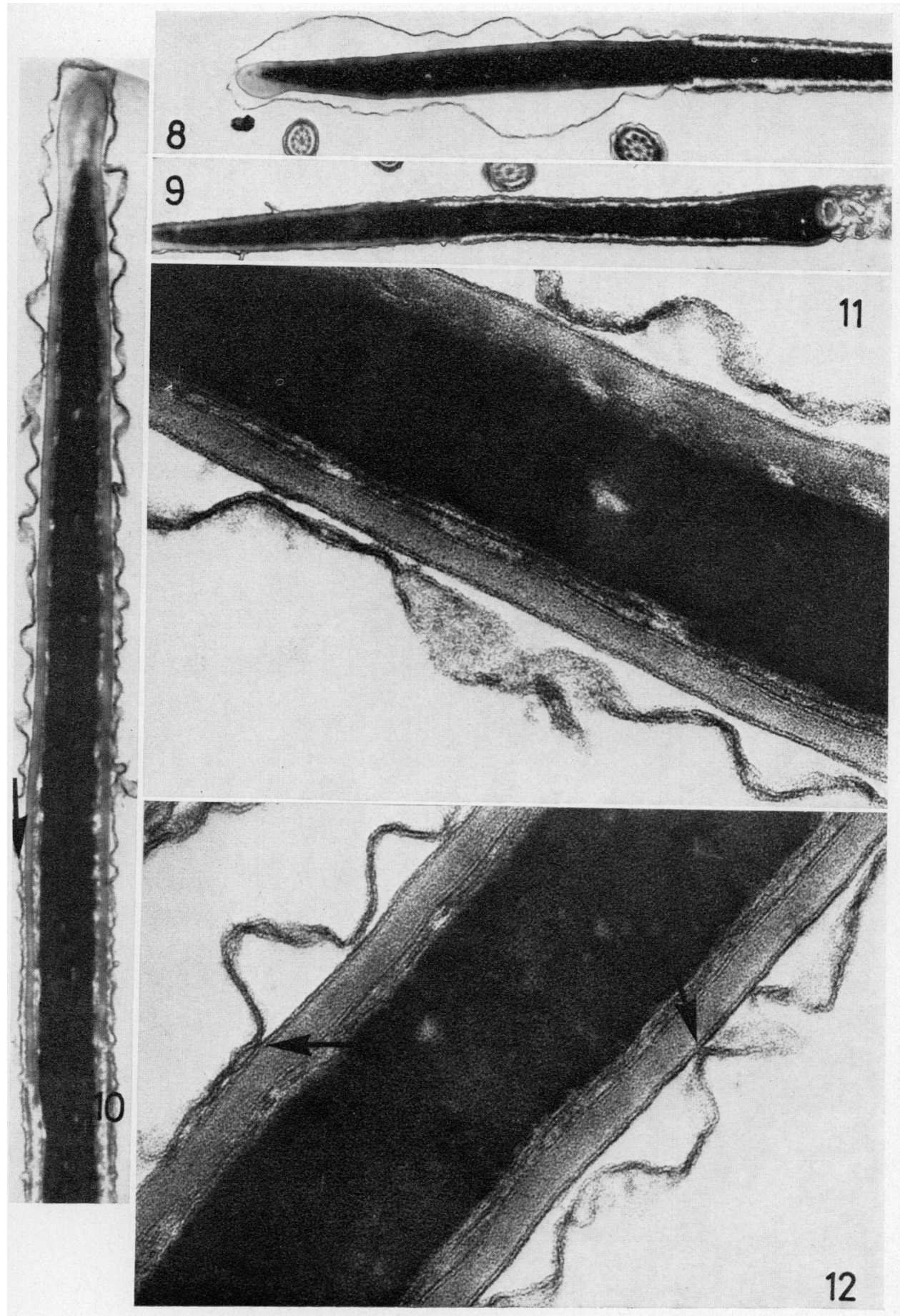


PLATE 4

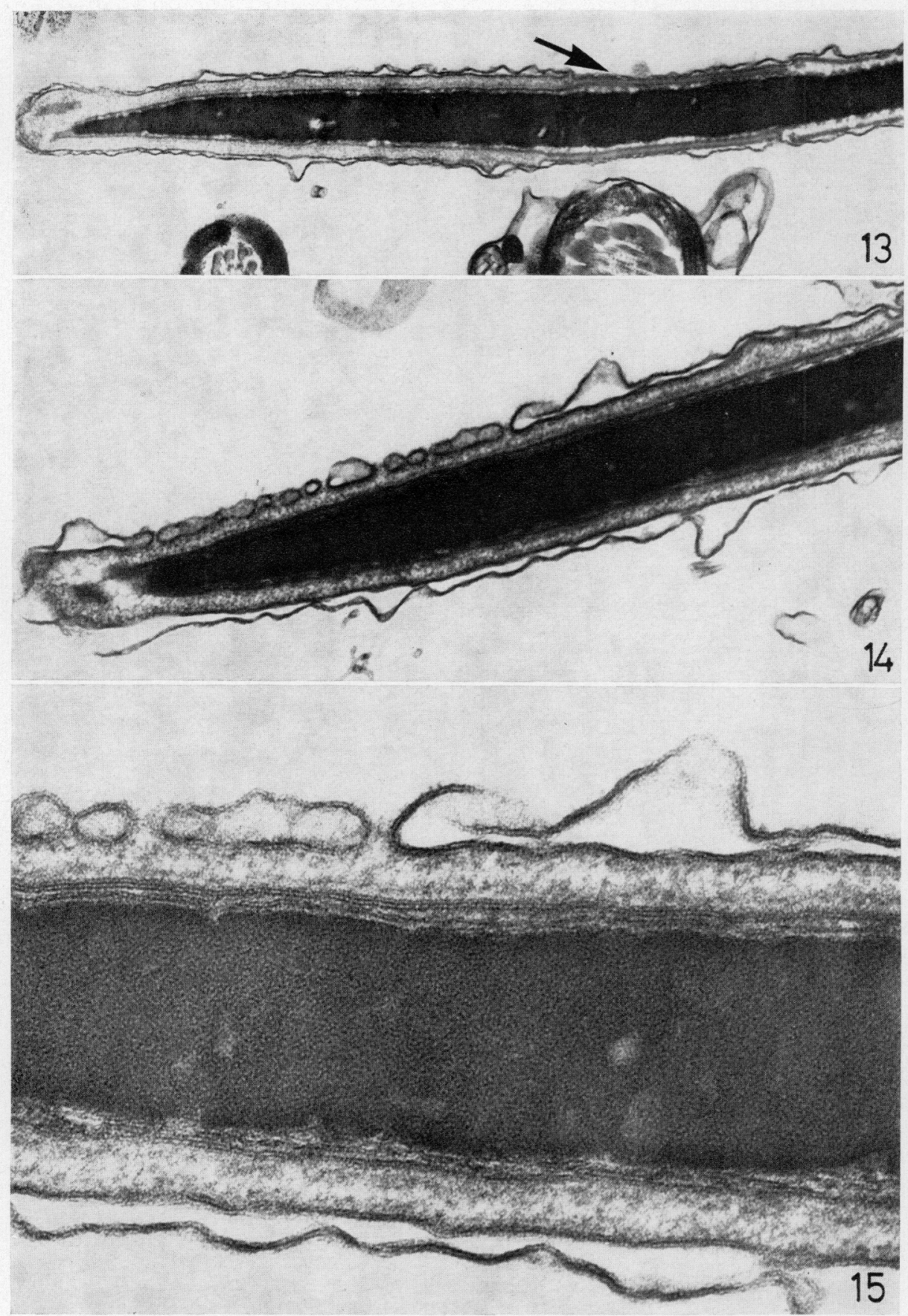


PLATE 5

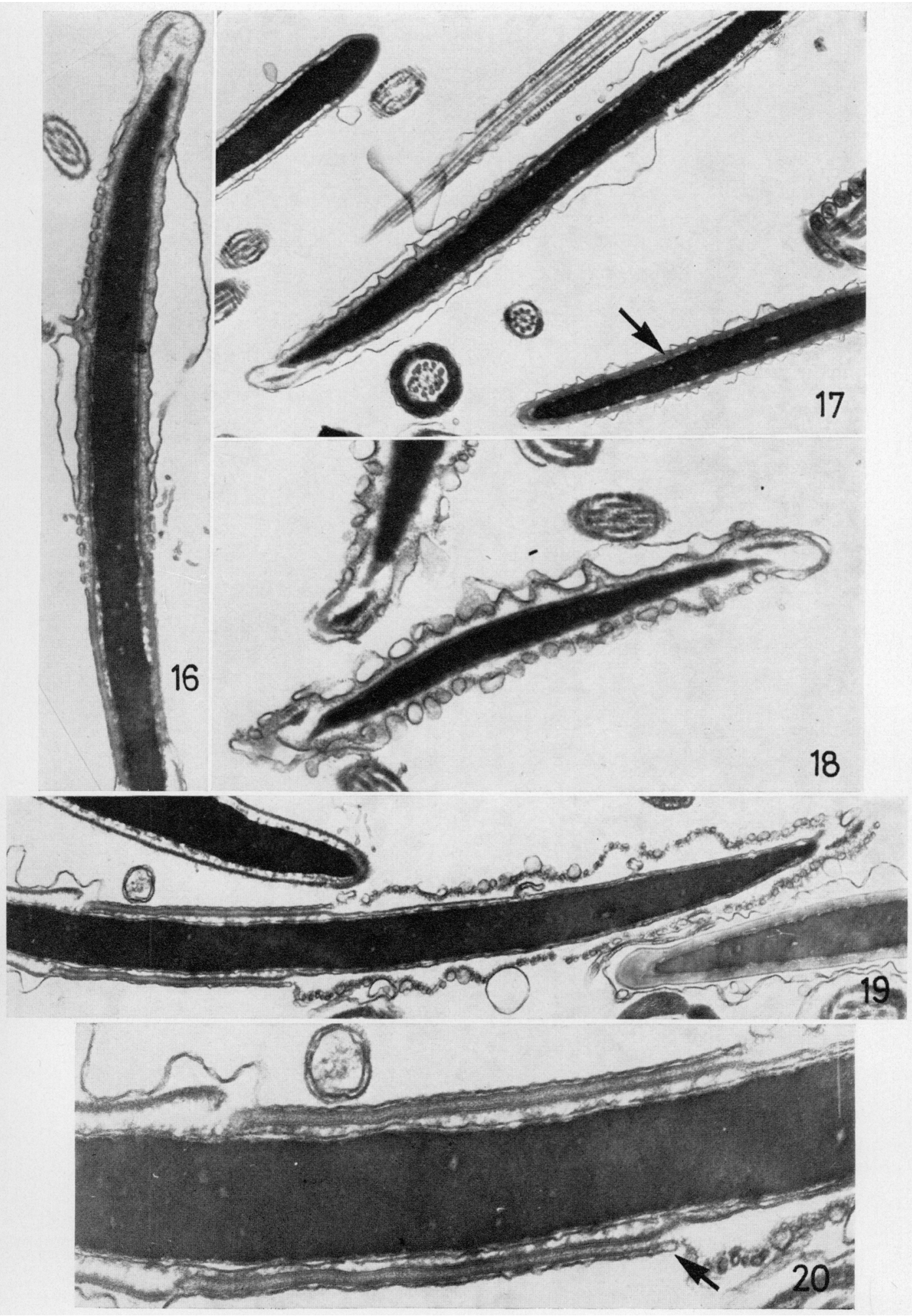


some reaction may vary considerably in size, but they usually lie within the range 50 to $500 \mathrm{~nm}$ (Pl. 2, Figs 5 and 7).

Thin transverse sections through a tubular meshwork would also appear vesicular. However, sections which include the surface plane of an a.r. spermatozoon clearly show that the plasmalemma and outer acrosome membranes have been replaced by small vesicles (Pl. 2, Figs 6 and 7). Transverse sections indicate that the vesicles remain as a cap around the head of the spermatozoon because of a meshwork of fibrillar material derived from the acrosome content to which they adhere (Pl. 2, Fig. 5). This material is sufficient to hold the vesicles even when the vesicular cap has been lost from the spermatozoon as it is in a few cases.

It has not been possible, as yet, to modify the conditions of the Hyamine incubation to produce a gradual increase in the percentage of spermatozoa showing the acrosome reaction. However, there are some spermatozoa at the earliest stages examined, between 15 and $60 \mathrm{sec}$ of incubation, which show a partial vesiculation. This partial vesiculation has never been found in more than $5 \%$ of the sample, and such spermatozoa have not been found after incubation with Hyamine for more than $5 \mathrm{~min}$. They may, therefore, indicate the course of the reaction, rather than being examples of partly blocked vesiculations.

Hyamine at $0.01 \%$ for $1 \mathrm{~min}$ does not seem to have any dramatic effect on the gap between the sperm plasmalemma and the acrosome outer membrane. A wide but variable gap between the two membranes (Pl. 3, Fig. 8) is found in 85 to $95 \%$ of unincubated spermatozoa, as reported in an earlier study (Wooding \& O'Donnell, 1971). Only 5 to $10 \%$ have a narrow gap between the membranes which are usually almost parallel (Pl. 3, Fig. 9). After brief Hyamine incubation such spermatozoa are virtually absent but there is now a small percentage, much less than $5 \%$, with a membrane showing a small period ruffling or corrugation (Pl. 3, Fig. 10). This low-amplitude ruffling of a closely applied plasmalemma appears to be specific to sperm populations treated briefly with low concentrations of Hyamine and seems also a likely prerequisite of the vesiculation reaction.

A connection between low-amplitude ruffling and vesiculation is borne out by the observation that the plasmalemma over the equatorial segment, which does not vesiculate, remains virtually flat yet very close to the equatorial segment outer membrane (Pl. 1, Figs 3 and 4; Pl. 3, Fig. 10). The ruffled plasmalemma does not normally come closer than 10 to $20 \mathrm{~nm}$ to the acrosome outer membrane (Pl. 3, Fig. 11) but indications of point membrane contacts equivalent to that in a tight junction have been observed (Pl. 3, Fig. 12).

In one or two of the ruffled spermatozoa the acrosome content is significantly less dense than in a normal, intact spermatozoon, and the apical crescent much more obvious (Pl. 4, Fig. 13). In partly vesiculated spermatozoa most show the ruffling of the plasmalemma where the two membranes have not yet fused (Pl. 4, Figs 14 and 15) although examples where this is not the case have occasionally been observed (Pl. 5, Fig. 16). In all of the partly vesiculated spermatozoa observed, the acrosome content is swollen and less dense than normal (Pl. 4, Fig. 14; PI. 5, Figs 16 and 17), but the equatorial segment which does not vesiculate remains unchanged. This suggests that changes induced in the 
acrosome content by the initial vesiculation may be important in completing the fusion of the membranes. The outer membrane of a partly vesiculated acrosome is not always smooth but may show signs of ruffling, bringing it closer to the overlying plasmalemma. Thus both membranes would be involved prior to the complete vesiculation (Pl. 5, Figs 17 and 18). Some of the vesicles produced may be much larger than the average, with a much greater contribution from the plasmalemma than from the acrosome membrane, indicating that the reaction can accommodate a plasmalemma considerably greater in area compared with the membrane with which it fuses.

What evidence there is suggests that Hyamine has two effects. It may strip the plasmalemma from the sperm head or induce the ruffling followed rapidly by a vesiculation reaction. Both processes are rapid, occurring within $60 \mathrm{sec}$. The speed of the vesiculation reaction produced by Hyamine plus the fact that partial vesiculations are found so rarely suggests a process analogous to the shattering of an area of toughened car winsdcreen induced from a single point. The lines of fracture in the glass would correspond to the lines of fusion between the membranes. On this hypothesis the observed change in the content of the acrosome might play a part in maintaining the impetus of the fusion once triggered by the contact of plasmalemma and acrosome membranes. It is certainly true that the acrosome equatorial segment neither changes in density nor does its outer membrane fuse with the plasmalemma, even though the membranes are closer in this region than over the upper acrosome.

\section{Physiological significance of the Hyamine acrosome reaction}

Morphologically, a very similar vesiculation reaction to that described above has been induced in bovine spermatozoa by incubating them in bovine uterine fluid without Hyamine (Pl. 5, Figs 19 and 20). After $3 \mathrm{hr}$ at $37^{\circ} \mathrm{C}$ about $40 \%$ of spermatozoa showed the reaction, with $35 \%$ intact and $25 \%$ swollen. Other bovine fluids had no effect (Table 2). No partial vesiculations were found. The similarity of the reactions induced by Hyamine and uterine fluid does suggest that the two processes are at least analogous, even though it is certainly not established that the underlying molecular mechanisms are identical. The variability in the time required for incubation (from 3 to $4 \mathrm{hr}$ ) to produce the vesiculation using uterine fluid has so far made observations of the earliest stages of the reaction even more difficult to find than when using Hyamine.

Table 2. Effect of various media on bovine sperm head morphology

\begin{tabular}{lccccc}
\hline \multirow{2}{*}{ Fluid } & Time of incubation & \multicolumn{3}{c}{ Sperm head morphology $(\%)$} \\
\cline { 3 - 6 } & $($ min $)$ & Intact & a.r.* & Swollen \\
\hline No incubation & - & 80 & 0 & 20 \\
Uterine fluid & 60 & 70 & 1 & 30 \\
& 180 & 39 & 40 & 21 \\
Follicular fluid & 270 & 72 & 35 & 32 \\
Heated $\dagger$ or unheated serum & 60 & 60 & 4 & 36 \\
\hline
\end{tabular}

* Spermatozoa with complete or partial acrosome reaction.

+ At $60^{\circ} \mathrm{C}$ for $30 \mathrm{~min}$. 
Considering the Hyamine fusion system again, there are some indications that it is a system working within physiological parameters. Although the vesiculation does not seem to require the presence of calcium it is blocked by incubation with azide and does not seem to occur in non-motile spermatozoa.

Sperm populations incubated in $0.01 \%$ Hyamine for less than $5 \mathrm{~min}$ do have about $50 \%$ of the motility of the original sample. It is possible, therefore, that those spermatozoa showing vesiculation are motile, although attempts to separate this group have been unsuccessful, probably because of the difficulty of getting rid of the Hyamine fast enough. The partial or complete loss of the plasmalemma behind the acrosome which occurs progressively during further incubation would certainly have a very drastic effect on motility. The vesiculation induced by Hyamine $(0.01 \%, 1 \mathrm{~min})$ may thus be a useful model for investigating the early membrane changes before fertilization. In those spermatozoa that undergo the reaction, the plasmalemma has to be close enough to the acrosome outer membrane and in the correct molecular configuration to fuse with it. There is some evidence that the Hyamine induces the ruffling of the plasmalemma and also closer contact between the ruffles and the acrosome membrane, but the mechanism by which it does so is obscure. Freeze-etch studies would seem to offer the best sort of information, when the early stages of vesiculation can be obtained.

A recent study has demonstrated that fusion may be preceded by an alteration of membrane contour, e.g. the protrusions induced from secretory granules in rat parotid gland follow a secretion stimulus (Schramm, Selinger, Salomon, Hytan \& Batzi, 1973). The early changes in the membranes in the acrosome reaction would be analogous to this process. However, such gross membrane changes give no information about the molecular mechanism of the process but merely make the fusion more likely to occur. A recent freeze-etch study has shown in Tetrahymena that there are preformed matching aggregates of intramembrane particles in membranes which are predictably about to fuse (Satir, Schooley \& Satir, 1973). The mechanism of the fusion process seems to depend on the association and complementary movement of these particles in the fusing mucocyst and cell boundary membranes.

Freeze-etch studies on bull spermatozoa (Koehler, 1966) show no differential distribution of intra-membrane particles in the head plasmalemma, although the detergent studies reported here (and Wooding, 1973) show this membrane to be very different in lability in different areas. It would thus be of considerable interest to have freeze-etch pictures of the plasmalemma of spermatozoa just before the acrosome reaction or of partly vesiculated spermatozoa. Such information may indicate more fully the details of the fusion process.

\section{REFERENCES}

Austin, G. R. (1967) Capacitation of spermatozoa. Int. F. Fert. 12, 25-31.

Barros, C., Bedford, J. M., Frankin, L. H. \& Austin, C. R. (1967) Membrane vesiculation as a feature of the mammalian acrosome reaction. 7. Cell Biol. 34, G1-C5.

BEDFord, J. M. (1970) Sperm capacitation and fertilisation in mammals. Biol. Reprod., Suppl. 2, 128158.

BEDFORD, J. M. (1972) Sperm transport capacitation and fertilization. In Reproductive Biology, Chapter 6, pp. 338-392. Eds. H. Balin \& S. Glasser. Excerpta Medica, Amsterdam. 
Buthala, D. A., Ericsson, R. J. \& Chubb, G. T. (1971) Interaction of Sendai virus and rabbit sperm. Biol. Reprod. 5, 325-329.

DAN, J. C. (1970) Morphogenetic aspects of acrosome formation and reaction. Adv. Morphogen. 8, $1-39$.

Gabara, B., Gledhill, B. L., Groce, C. M., Cesarini, J. P. \& Koprowski, H. (1973) Ultrastructure of rabbit spermatozoa after treatment with lysolecithin and in the presence of hamster somatic cells. Proc. Soc. exp. Biol. Med. 143, 1120-1124.

Hartree, E. F. \& Srrvastava, P. N. (1965) Chemical composition of the acrosomes of ram spermatozoa. F. Reprod. Fert. 9, 47-50.

Koemler, J. K. (1966) Fine structural observations in frozen etched bovine spermatozoa. J. Ultrastruct. Res. 16, 359-375.

Multamaki, S. \& Pelliniem, L. J. (1973) Ultrastructure of subcellular fractions of bull spermatozoa after Hyamine treatment. Z. Zellforsch. mikrosk. Anat. 144, 395-408.

O'DonNELl, J. M. (1969) Intracellular levels of sodium and potassium in bull spermatozoa in relation to cell metabolism. 7. Reprod. Fert. 19, 207-208.

O'Donnell, J. M., Symons, D. B. A. \& Wooding, F. B. P. (1970) Immunofluorescence and electron microscopy of acrosomes of bull spermatozoa. F. Physiol, Lond. 210, 120p-123p.

Poste, G. \& Allison, A.G. (1973) Membrane fusion. Biochim. biophys. Acta, 300, 421-465.

SatiR, B., Schooley, C. \& SATIR, P. (1973) Membrane fusion in a model system. Mucocyst secretion in Tetrahymena. J. Cell Biol. 56, 153-176.

Schramm, M., Selinger, Z., Salomon, Y., Hytan, H. \& Batzi, S. (1973) Pseudopodia formation by secretory granules. Nature, Lond. 240, 203-205.

Wooding, F. B. P. (1973) The effect of Triton X100 on the ultrastructure of ejaculated bovine sperm. $\mathcal{F}$. Ultrastruct. Res. 42, 502-516.

Wooding, F. B. P. \& O'DonNeLl, J. M. (1971) A detailed ultrastructural study of the head membranes of ejaculated bovine sperm. F. Ultrastruct. Res. 35, 71-85. 